Supporting Information

\title{
Atomic-Resolution Topographic Imaging of Crystal Surfaces
}

Ryo Ishikawa ${ }^{1,2, *}$, Riku Tanaka ${ }^{1}$, Kazuaki Kawahara ${ }^{1}$, Naoya Shibata ${ }^{1,3}$, and Yuichi Ikuhara ${ }^{1,3}$

${ }^{1}$ Institute of Engineering Innovation, University of Tokyo, Bunkyo, Tokyo, 113-8656, Japan

${ }^{2}$ PRESTO, Japan Science and Technology Agency, Kawaguchi, Saitama 332-0012, Japan

${ }^{3}$ Nanostructures Research Laboratory, Japan Fine Ceramics Center, Nagoya, Aichi 456-8587, Japan

*e-mail: ishikawa@sigma.t.u-tokyo.ac.jp (R.I.)

\section{ROI-size dependence of the contrast profile}

Since the depth profile may depend on the size of region-of-interest (ROI), we investigate the ROI-size dependence of (a) mean value $(\mu)$, (b) standard deviation $(\sigma)$, and (c) contrast $(\sigma / \mu)$, respectively. The depth profiles obtained from $\mathrm{Sr}$ atomic column as a function ROI-size are given in Figure S1, where we use the simulated focal-series ADF STEM images with the thickness of $10.2 \mathrm{~nm}$. For convenience, the radii of the ROI-size are given by the function of $\sigma_{\mathrm{g}}$ : the standard deviation of the fitted $2 \mathrm{D}$ gaussian for the $\mathrm{Sr}$ atomic column at zero defocus. The $2 \mathrm{D}$ gaussian function may be expressed as

$$
G(x, y)=A_{g} \exp \left(-\frac{\left(x-x_{0}\right)^{2}+\left(y-y_{0}\right)^{2}}{2 \sigma_{g}^{2}}\right),
$$

where $A_{g}$ is an amplitude and the atom is located at $\left(x_{0}, y_{0}\right)$. As shown in Fig. S1(a), the depth profile of the mean value (mean profile) is maximized at slightly under-defocus condition with the smaller ROI-size. This is probably the reason the most of microscopists select slight under-defocus condition as an optimum defocus, by looking at atomic columns. As increasing ROI-size, the maxima of mean profiles are gradually shift toward under-defocus, and it is therefore that the mean profile is not suitable to determine the surface atomic structure. While, in the profiles of standard deviation and contrast of Fig. S1(b) and (c), these depth profiles are well maximized at almost zero-defocus, and moreover, the ROI-size dependence is negligibly small. Compared with the profiles of the standard deviation, the contrast profiles have more symmetric at the zero-defocus and the peak height is higher than those in standard deviation. When we use a relatively small ROI-size such as $2 \sigma_{\mathrm{g}}$, the peak position in the contrast profile is slightly shift toward under-defocus (a few Ångstrom). However, with the ROI-size larger than $3 \sigma_{\mathrm{g}}$, the peak positions are well maximized at zero defocus, and therefore we select the ROI-size smaller than $3 \sigma_{\mathrm{g}}$ than can be negligible of the ROI-size effect (typically we use $3 \sigma_{\mathrm{g}}$ ). We note that the standard deviation is related to the sharpness of the atomic column rather than the absolute intensity, and hence the smaller and less bright atomic column would have the higher standard deviation. It is also noteworthy that the peak position in a contrast profile does not necessary to be maximized exactly at zero defocus, because we will relatively determine the surface height at respective atomic columns. However, it is necessary that the peak positions in the contrast profiles should 
be the same with different specimen thicknesses (in a target specimen thickness range), which can be found in Figure 1.

\section{The effects of incoherent electron sources and quantum noise on the contrast profile}

In comparison with the simulated contrast depth profiles in Figure 1, the experimental depth profiles in Fig. $3 \mathrm{~b}$ shows more symmetric. To investigate the present discrepancy, we here consider three effects of (1) effective source size, (2) chromatic aberration, and (3) quantum noise. Figure S2(a) show the simulated focal-series of ADF STEM images: (i) as-simulated, (ii) with effective source size, (iii) with effective source size and chromatic aberration, (iv) with effective source size, chromatic aberration and quantum noise, respectively. To include finite source size, the simulated images are convolved the 2D gaussian function with full-width-half-maximum of $0.4 \AA$. The chromatic aberration coefficient and the energy spread of our gun are $C_{c}=1.75 \mathrm{~mm}$ and $\Delta E=0.34 \mathrm{eV}$, respectively. ${ }^{1}$ For the chromatic aberration effect, the simulated aberration-free focal-series images are convolved with the focus spread of $\Delta f=C_{c}\left(\Delta E / E_{0}\right){ }^{2}$ According to our experimental dose condition of Fig. 3(a), the quantum noise (Poisson noise) is added with the electron dose condition of $217 \mathrm{e}^{-} / \mathrm{pix}$ (acquisition time: $4 \mu \mathrm{s} /$ pix, probe current: $8.7 \mathrm{pA}$ ). The selected focal-series ADF STEM images (specimen thickness: $15.2 \mathrm{~nm}$ ) are shown in Fig. S2(a). Compared with the as-simulated images, the $\mathrm{Sr}$ atomic columns become broader by effective source size and chromatic aberration. Figure S2(b) shows the Sr contrast profiles obtained from respective focal-series images of Fig. S2(a). Although the peak positions are not affected by the finite source size and chromatic aberration, the peak profiles become broader and more symmetric. In the noise-added simulation, the peak position may be slightly shifted, and the peak profile shows more symmetric. However, the peak position can be retrieved by our statistical approach. To avoid beam irradiation damages, the experiments were performed at relatively low dose condition, which introduces large amounts of intensity variation. Therefore, the standard deviation within the ROI is significantly increased, especially out-of-focus condition. Clearly, the peak height in the noise-added contrast profile becomes nearly half of the other profiles, suggesting that the standard deviation is increased at the out-of-focus condition. Consequently, the disappearance of the tails in experimental contrast profiles can also be explain by the quantum noise. 
Supporting Information

\section{Rigid registration vs. non-rigid registration}

Although the ROI-size effect on the contrast profile is negligibly small, the location of the ROI is a nonnegligible parameter. In a focal-series ADF STEM images, most of the images are acquired at out-ofdefocus condition, and it is difficult to accurately identify all the atom locations (1,518 atomic columns) as per frame. For the determination of the atomic column positions, we use the averaged focal-series ADF STEM images (Fig. 3a) by 2D gaussian fitting, and the locations of all the ROIs are fixed for all the frames. Under the analysis condition, it is prerequisite to accurately align all the frames, and hence non-rigid registration is more suitable for this purpose. ${ }^{3}$ Figure S3 shows the contrast profiles obtained from a $\mathrm{Sr}$ atomic column with rigid and non-rigid registrations, respectively. Although these contrast profiles seem to be very similar, the peak-to-background ratio (PB-ratio) with non-rigid registration is approximately $15 \%$ better than that in rigid registration. This result suggests that the mis-location of ROI is a main issue to reduce the signals in contrast profiles, and hence we adopt non-rigid body registration rather than rigid registration. It is noteworthy that, with non-rigid-body registration, the intensities might be slightly and locally changed. However, there are more than 300 pixels within a single ROI, and the intensity redistributed should not be significant in our analysis.

\section{Peak deconvolution of total PDF}

In our classification of atomic-layer-heights, peak deconvolution of total PDF (probability density function) is a key process, and we here describe our procedure to determine the number of peaks. As shown in Fig. $3 \mathrm{~d}$ and 3e, there are 5 and 4 distinct peaks in respective total PDFs. Considering into the depth range of $\sim 2 \mathrm{~nm}$ and the lattice parameter of $\mathrm{SrTiO}_{3}(0.39 \mathrm{~nm})$, it is reasonable to assume that these total PDFs may be deconvolved into $4-6$ peaks. In our deconvolution, we use the model function as mixed gaussian profiles (Eq. (4) in Methods):

$$
G\left(z ; w_{m}\right)=\sum_{m=1}^{M} a_{m} \cdot \exp \left(-\frac{\left(z-z_{m}\right)^{2}}{2 \sigma_{m}^{2}}\right),
$$

where $m$ is the index for gaussian profiles $(m=1,2, \ldots, M), a_{m}, z_{m}$ and $\sigma_{m}{ }^{2}\left(w_{m}=\left\{a_{m}, z_{m}, \sigma_{m}\right\}\right)$ for each $m$ are the amplitude, peak position, and variance, respectively. To determine the number of gaussian profiles of $M$, we examined the cases of $M=4,5,6$ for respective total PDF profiles. The fitting degree is evaluated by the sum of squares of residual (chi-squared test):

$$
\chi^{2}=\sum_{z}\left(P D F(z)-G\left(z ; w_{m}\right)\right)^{2},
$$

where $z$ is depth. To globally explore an optimum fitting parameter set of $w_{m}$, we perform peak deconvolution by random walk Metropolis-Hastings (RWMH) algorithm. Figure S4(a) and 4(b) show the histograms of the chi-squared test accepted in the Metropolis tests with $M=4,5,6$, for $\mathrm{Sr}$ and Ti-O atomic columns, respectively. In general, the goodness of fit may be improved by increasing the number of fitting 
parameters. This holds true in both cases of Fig. S4(a) and 4(b), and these chi-squared tests are minimized at 6 gaussian profiles. However, the difference of chi-squared tests between 5 and 6 gaussian profiles are considerably small, and hence the fitting with 6 gaussian profiles may be overfitting. Fig. S4(c) shows the total PDF and the deconvolved 5 and 6 gaussian profiles for Sr atomic column, respectively. With 6 gaussian profiles, there is a negative gaussian profile (no physical meaning), suggesting it should be overfitting. Thus, the number of gaussian profile for Sr atomic column is determined to be 5. Fig. S4d shows the total PDF and the deconvolved 5 and 6 gaussian profiles for Ti-O atomic column. As indicated by arrowheads, there are evident 4 peaks in the total PDF at Ti-O atomic column. The rightmost peak is considerably large, and it is reasonable to deconvolve the peak into 2 gaussian profiles. The other three peaks are also well reproduced by three gaussian profiles, and overall, the total PDF is well reproduced by 5 gaussian profiles. With 6 gaussian profiles, a new gaussian profile \#3 appears, but there is no peak in the total PDF. Owing to the gaussian profile \#3, the distance between \#1 and \#2 becomes considerably close, which may be physically inappropriate, i.e., overfitting. Therefore, we conclude that both the total PDFs of $\mathrm{Sr}$ and Ti-O atomic columns are composed of 5 gaussian profiles, respectively.

\section{Stability of the microscope and stability of the sample under the beam irradiation}

Although we could not directly measure the drift along the depth, it could be a similar level to that of inplane drift. However, in addition to the stage instability, electron probe instability may also be affected to the drift along the depth. To investigate the stability of our microscope, we have time-sequentially acquired 100 frames of ADF STEM images obtained from a flat surface area, with a fixed defocus condition (near zero-focus), where the acquisition time and the beam current were $4 \mu \mathrm{s} /$ pix and $10.7 \mathrm{pA}$, respectively. The total in-plane drift for whole frames was $7.4 \AA$ (drift rate in average is $7.4 \mathrm{pm} / \mathrm{frame}$ ), which is a similar level to the main experiment of Fig. 3, and the drift along the depth is also expected to be the same level. With this assumption, the contrast change of a single atomic column should be almost constant over the frames, if the electron probe is stable enough. Figure S5(a) and (b) show a part of the frame averaged ADF STEM image and respective 100 frames of the single $\mathrm{Sr}$ atomic column, as marked by the white square in Fig. S5(a). Fig. 5(c) shows the contrast as a function of frame, obtained from the single Sr atomic column (blue dots), where the focal-series contrast profile (red dots, Sr atomic column) is also plotted as a reference (the same data in Fig. S3). Compared with the focal-series contrast profile, the defocus-fixed contrast values are almost constant as a function of frame. The observed small contrast reduction as a function of frame can be explain by the similar level of the stage drift along the depth. In addition, no significant geometric aberrations were introduced during time-sequential acquisition. We conclude that our electron probe should be much stabler than the stage drift. We note that there are small amounts of lower orders of residual aberrations and specimen tilt, which may affect to determine the surface height. However, these effects 
could be equally contributed to all the atomic columns, and equally shift the maximum contrast defocus for all the atomic columns. But if the aberrations are changing as a function of time, it may be affected our reconstruction algorithm.

In our depth sectioning, we need to acquire relatively a large number of images as a function of defocus, and hence we must care of surface damaging of the sample. To reliably avoid electron beam damage, we use relative low dose condition: the low beam current $(8.7 \mathrm{pA})$ and the fast scanning of the probe (4 $\mu \mathrm{s} / \mathrm{pix})$. We also realize that the heating of the sample is considerably helpful to avoid electron beam damage in our experiments, especially in the case of $\mathrm{SrTiO}_{3}$, although we still do not understand the mechanism (at higher temperatures, semiconducting materials become slightly conductive, which may help to avoid beam damages). To investigate the stability of our sample under the beam irradiation, we analyze the data set of time-sequentially acquired 100 images, as shown in Fig. S5(a). According to the previous reports, the mean value of ADF STEM image is sensitive to the number of atoms along the columns. ${ }^{4-6}$ We therefore check the local mean values as a function of frame, and the typical mean value profile is given in Fig. $5 \mathrm{~S}(\mathrm{~d})$, where the location of the ROI is marked in Fig. 5S(a). The observed mean values are basically constant as a function of frame, and the standard deviation is as small as $1.9 \%$ that should be originated to the quantum noise owing to the present low dose condition. It is noteworthy that the observation in main text is mostly out-of-focus condition, and it is therefore the observation condition in main text must be much milder than this in-focus experiment. We therefore conclude that our specimen surface could be considerably stable under the present experimental condition, especially in the experiment of the depth sectioning.

\section{The effect of Debye-Waller factors}

In our experiment, we performed ADF STEM imaging at $673 \mathrm{~K}$, and hence Debye-Waller factors may be affected on our surface reconstruction algorithm. To investigate the Debye-Waller effects on ADF STEM images, we performed image simulations with larger Debye-Waller factors. Since we could not find any reports on Debye-Waller factors of $\mathrm{SrTiO}_{3}$ at higher temperatures, we investigate the temperature effect on ADF STEM images as follow. In a semi-classical approximation, the thermal displacements of $\langle u\rangle$ is scaled with $\langle u\rangle=\left\langle u_{0}\right\rangle \sqrt{T / 300}$, where $T$ is temperature in Kelvin. ${ }^{7}$ We therefore perform focal-series ADF STEM image simulations with roughly 50\% larger Debye-Waller factors by referring the Debye-Waller factors at $296 \mathrm{~K}^{8}{ }^{8}$ Figure S6 shows the Sr contrast profile as a function of defocus with different specimen thicknesses. Although the profile is relatively noisy, the peak positions are almost at zero-focus for all the specimen thicknesses. Therefore, we conclude that the Debye-Waller factor effect could be negligible in our reconstruction algorithm. We note that the noisy contrast profile should be related to the small number of frozen phonon configurations. Typically, it is enough to use 20 frozen phonon configurations. However, 
the thermal displacement is 1.5 times larger than that at room temperature, it may be necessary to use much larger number of frozen phonon configuration $(>100)$ to obtain smooth contrast profile (the effect can be seen as non-circular symmetric atomic columns in the simulated images, except for the zero-defocus condition).

\section{Image simulations with the termination of $\mathrm{TiO}_{2}$ layer}

For $\mathrm{SrTiO}_{3}(001)$ surface termination, there are two possible charge neutral surfaces of (i) $\mathrm{SrO}$ and (ii) $\mathrm{TiO}_{2}$ layers. In main text, we show the image simulations with the termination of SrO layer, and we here perform the image simulation with the termination of $\mathrm{TiO}_{2}$ layer. Figure $\mathrm{S} 7$ shows the contrast profiles obtained from (a) Ti-O and (b) Sr atomic column, respectively. The contrasts at Ti-O atomic column are basically well maximized nearly at the entrance surface, and the contrasts at $\mathrm{Sr}$ atomic columns are evidently maximized at sub-surface. It is therefore possible to determine the entrance surface height in both $\mathrm{SrO}$ and $\mathrm{TiO}_{2}$ terminations.

\section{Fitting standard error maps for respective atomic columns}

In RWMH algorithm, it is possible to estimate the standard error of the fitting, corresponding to the standard deviations of respective PDF profiles, as shown in Fig. 3(c). Figure S8 shows the estimated standard errors of the fitting $\left( \pm \sigma_{\mathrm{z}}^{\text {fit }}\right)$ for (a) $\mathrm{Sr}$ and (b) Ti-O atomic columns, respectively. As shown in the histogram of Fig. 3(f), the standard errors for $96.2 \%$ of atomic columns have better than $\pm 0.9 \AA$. Overall, the thinner region has a smaller standard error. However, at the very thin region (arrows in Fig. S8), the contrast profile becomes noisy owing to the low dose observation, and the standard error becomes larger.

\section{Cleanness of the sample surface}

To show the cleanness of our sample surface, we acquired relatively low-magnified annular bright-field (ABF) STEM image of $\mathrm{SrTiO}_{3}$ viewed along the [001] direction, as shown in Fig. S9. In a similar manner of Fig. 2 in main text, there are two types of the surface facet planes of $\{100\}$ and $\{110\}$, respectively. Although ABF STEM imaging is quite sensitive to the low Z-number elements, we did not observe extra contrast on the surface, suggesting that our sample has no carbon-contaminations under the present in situ heating condition.

\section{Classification and precisions of atomic-layer-heights}

To classify the surface height into atomic-layer-heights, we performed peak deconvolution of the total PDFs, as shown in Fig. S4. However, there are unavoidable overlaps between the nearest deconvolved 
gaussian profiles, owing to the experimental noise. This can be found in the normalized gaussian profiles at $\mathrm{Sr}$ atomic column in Fig. S10(a):

$$
\widehat{G}\left(z ; w_{m}\right)=\frac{1}{\sqrt{2 \pi \sigma_{m}^{2}}} \exp \left(-\frac{\left(z-z_{m}\right)^{2}}{2 \sigma_{m}^{2}}\right),
$$

where the parameters are the same in Eq. (2). It is noteworthy that the overlaps between the second nearest gaussian profiles are negligibly small, and hence we only consider one atomic-layer-height misclassification. When the entrance height of an $i^{t h}$ atom column is $z_{i}$, (we here use the highest probability in the PDF), the probability belonging to the $m_{0}{ }^{\text {th }}$ layer may be evaluated as follows:

$$
\operatorname{Prob}\left(z_{i}, m_{0}\right)=\frac{\widehat{G}\left(z_{i} ; w_{m 0}\right)}{\sum_{m} \hat{G}\left(z_{i} ; w_{m}\right)} .
$$

As an example, we show the probability maps at Sr atomic columns in Fig. S10(b), where the surface heights are assigned to be layer-1. As indicated by the color bar, the red and blue colors correspond to the higher and lower probabilities. At the layer-1, the right-top region is covered by strong red colors, and the region could be a large terrace with relatively high probability $(>80 \%)$. In this terrace, there are some isolated point defects denoted as (a) $V_{\mathrm{Sr}}$ and (b) $V_{\mathrm{Sr}}$-cluster, respectively. The probabilities of these surface vacancies are (a) $84 \%$, (b) $72 \%$ (in average), respectively. The white colors correspond to that the probability is close to $50 \%$, and hence the confidence is too low to discuss surface point defects. Since the atomic height would be changed at the terrace edge, white atomic columns may appear near the edge region. For example, the probability in the layer-1 at (c) is 52\%, and thus the atomic column could be layer-2 (but not layer-3). It is noteworthy that the number of white atomic columns in layer 1 (probability: $45-55 \%$ ) is only 3.4\% (13 atomic columns), and hence most of atomic columns in layer-1 have relatively high reliability. At the terrace edge regions such as dotted lines or region (d), the surrounding atomic columns have strong red and blue colors, where the probability of the line step of (d) to be layer- 2 is $87 \%$ in average, and hence the formation of (0-11) surface is highly reliable.

\section{Analysis flowchart of our algorithm}

Figure S11 shows the analysis flowcharts of our algorithm: (a) overall flowchart, (b) profile fitting flowchart, and (c) RWHM fitting flowchart, respectively. The detailed information for each procedure such as 'extract depth profiles' or 'peak deconvolution' can be found in this Supporting Information. 

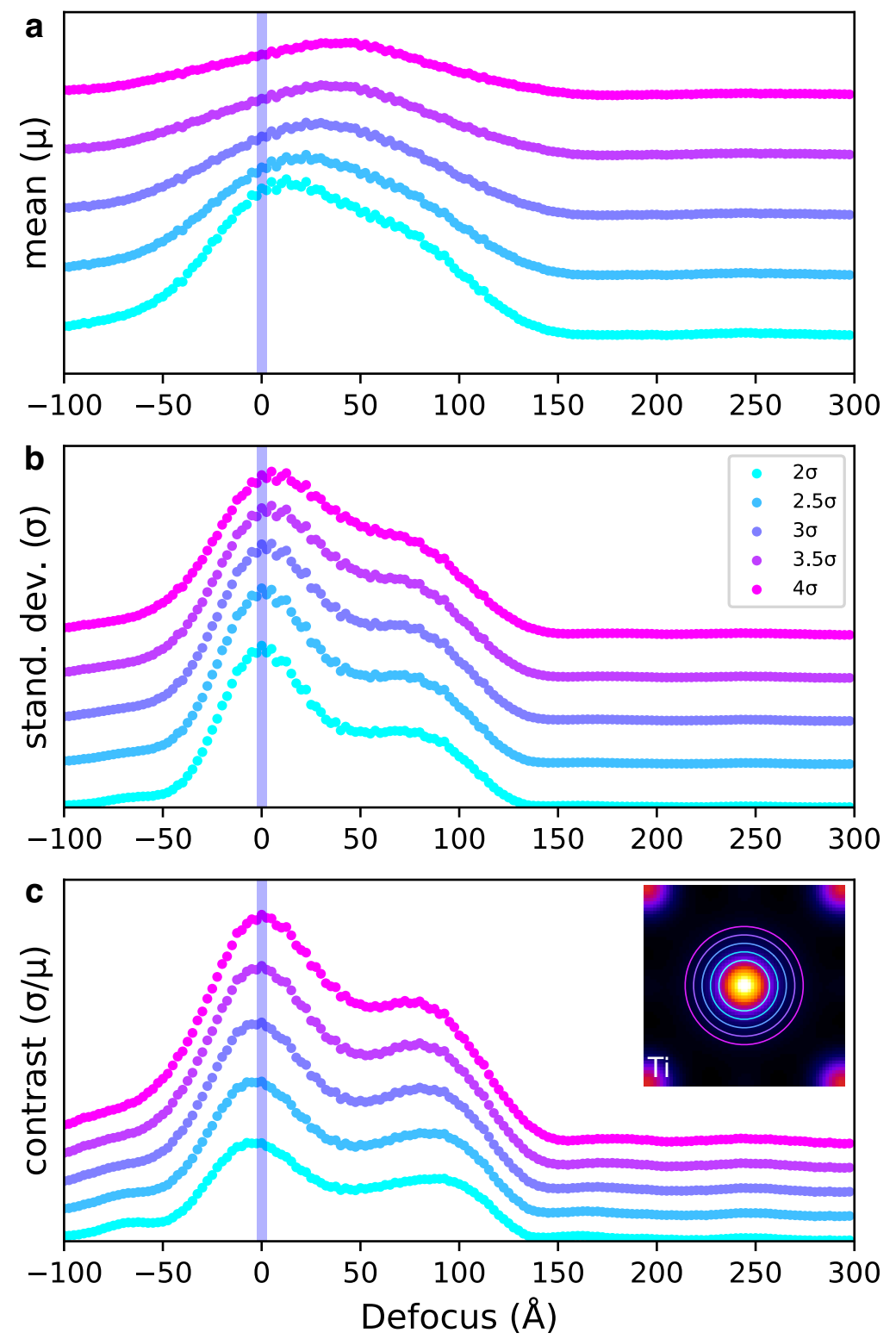

Figure S1. Depth profiles of (a) mean value $(\mu)$, (b) standard deviation $(\sigma)$, (c) contrast $(\sigma / \mu)$ as a function of ROI size at Sr atomic column, obtained from the simulated ADF STEM images with the thickness of $10.5 \mathrm{~nm}$. The radii of the ROI sizes are $2 \sigma_{\mathrm{g}}, 2.5 \sigma_{\mathrm{g}}, 3 \sigma_{\mathrm{g}}, 3.5 \sigma_{\mathrm{g}}, 4 \sigma_{\mathrm{g}}$, respectively, where $\sigma_{\mathrm{g}}$ corresponds to the standard deviation of the fitted 2D gaussian of the $\mathrm{Sr}$ atomic column at zero defocus and the respective ROIs are given in (c) (right top). 


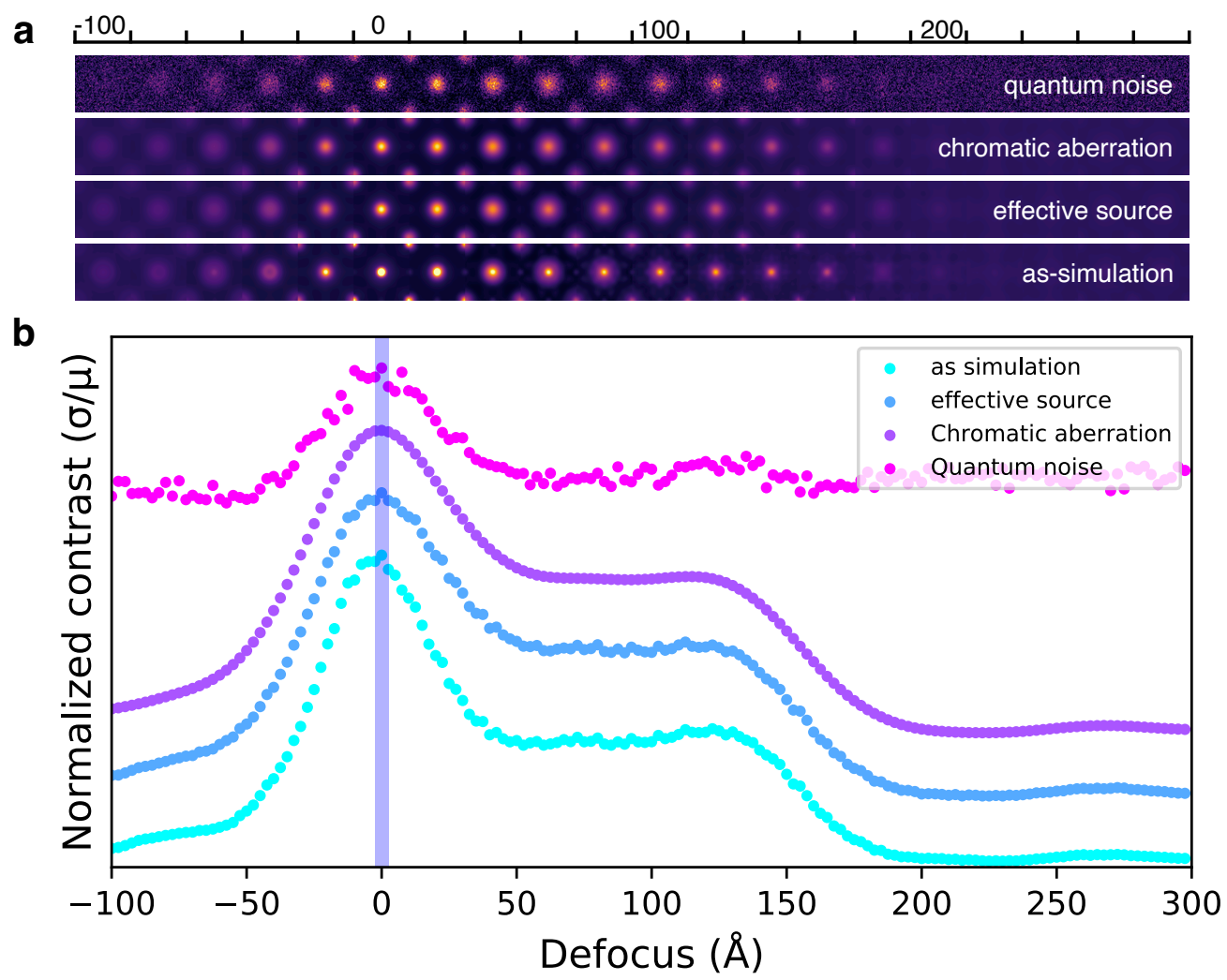

Figure S2. (a) Selected ADF STEM images, including the following effects from bottom to top: (i) assimulation, (ii) effective source size, (iii) effective source size and chromatic aberration, (iv) effective source size, chromatic aberration and quantum noise, respectively. (b) The Sr contrast profiles obtained from the corresponding simulation images (the assumed specimen thickness is $15.2 \mathrm{~nm}$ ). 
Supporting Information

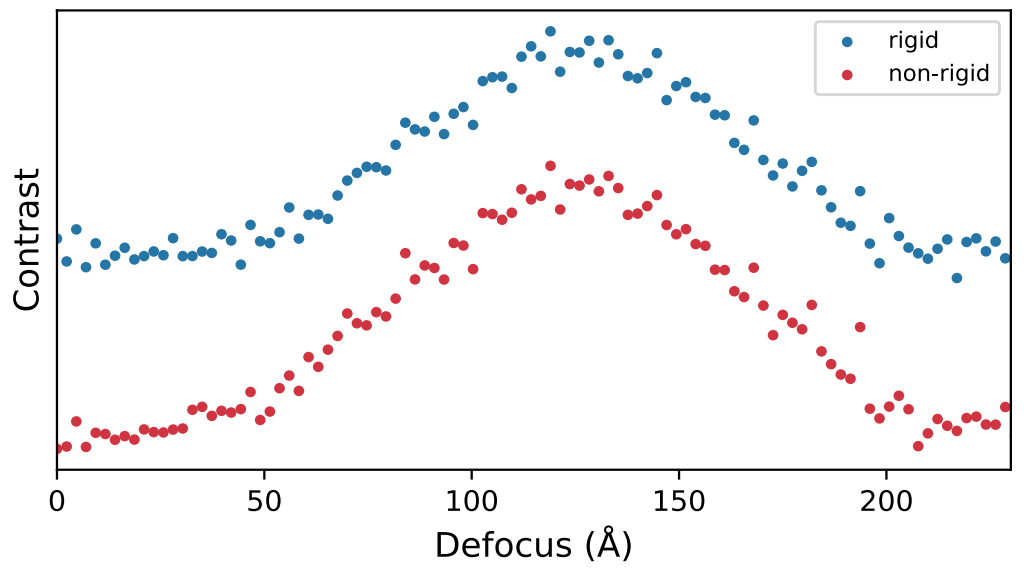

Figure S3. Contrast profile obtained from a Sr atomic column with different image alignment algorithms: (i) rigid registration and (ii) non-rigid registration, respectively. 
Supporting Information

a
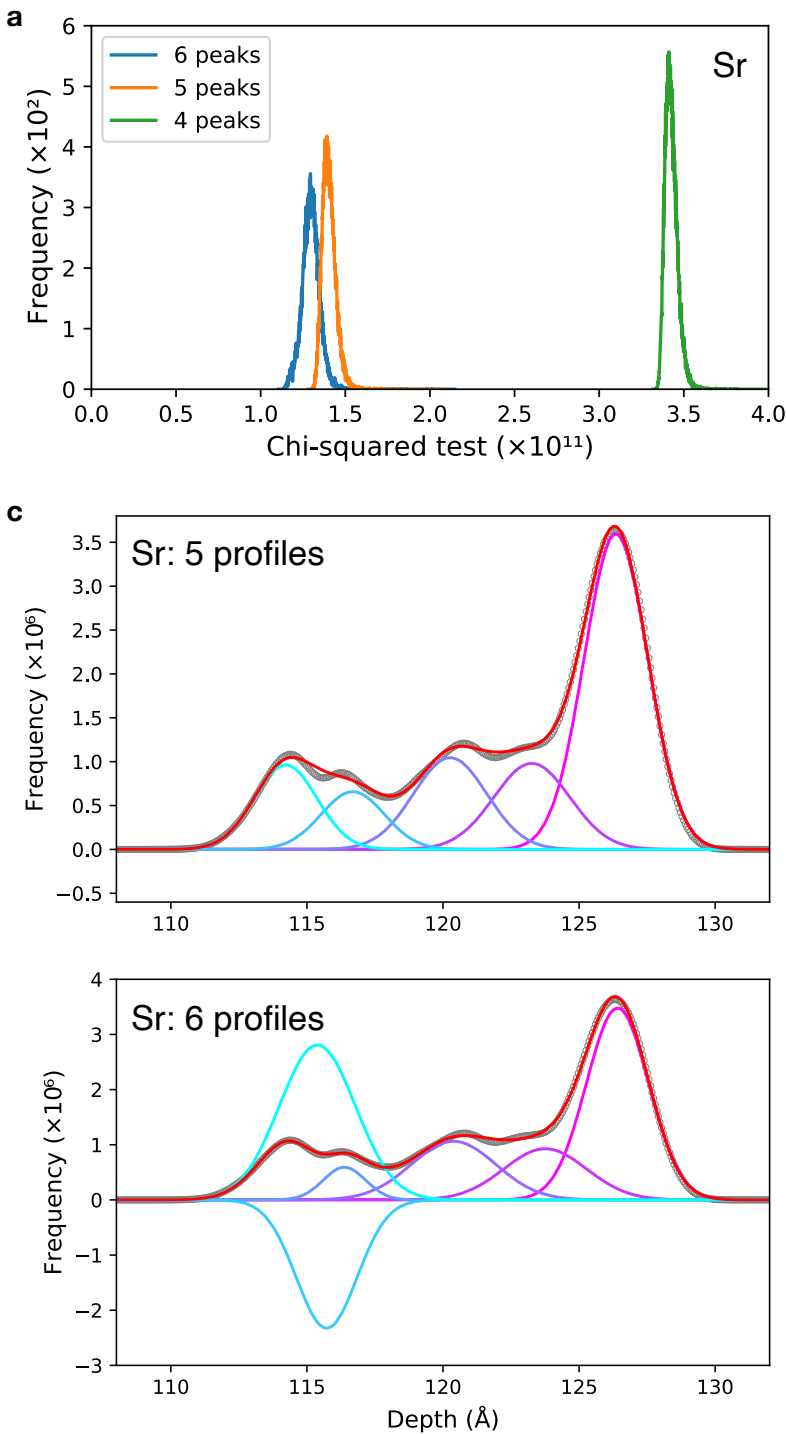
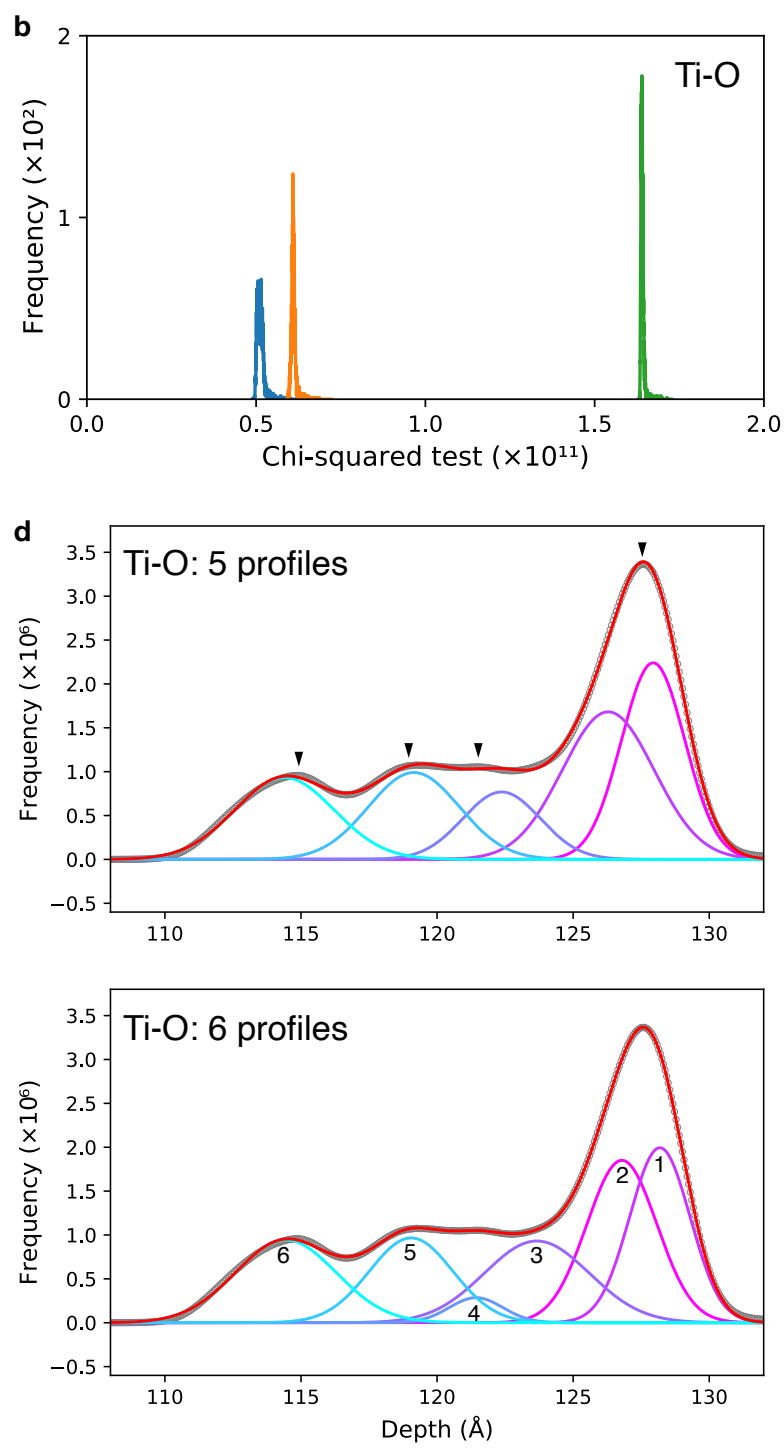

Figure S4. Chi-squared tests with different number of peaks (4, 5, and 6) for (a) Srand (b) Ti-O atomic columns, respectively. Total PDF profiles and deconvolved gaussian profiles for (c) $\mathrm{Sr}$ and (d) Ti-O atomic columns, where the number of deconvolved gaussian profiles are 5 and 6 , respectively. 


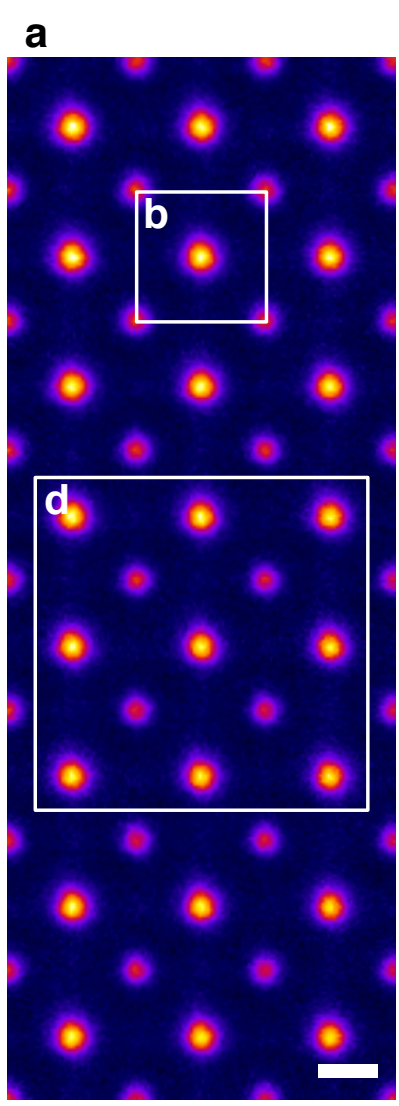

C

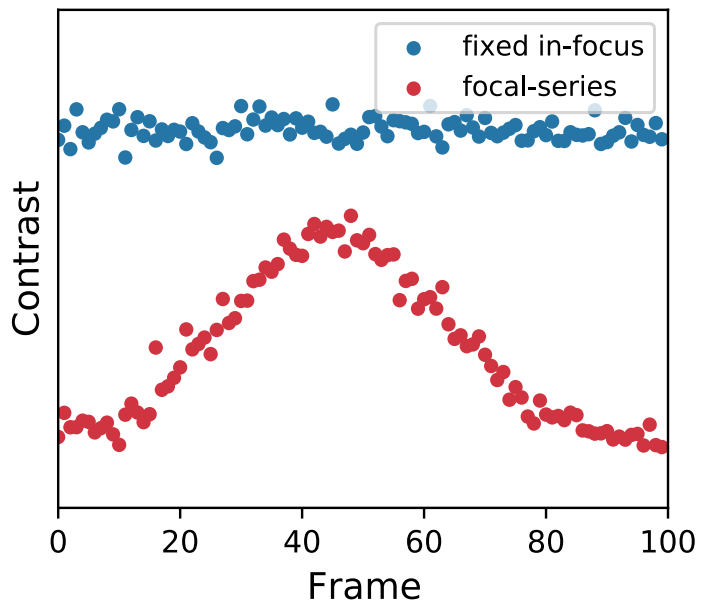

b

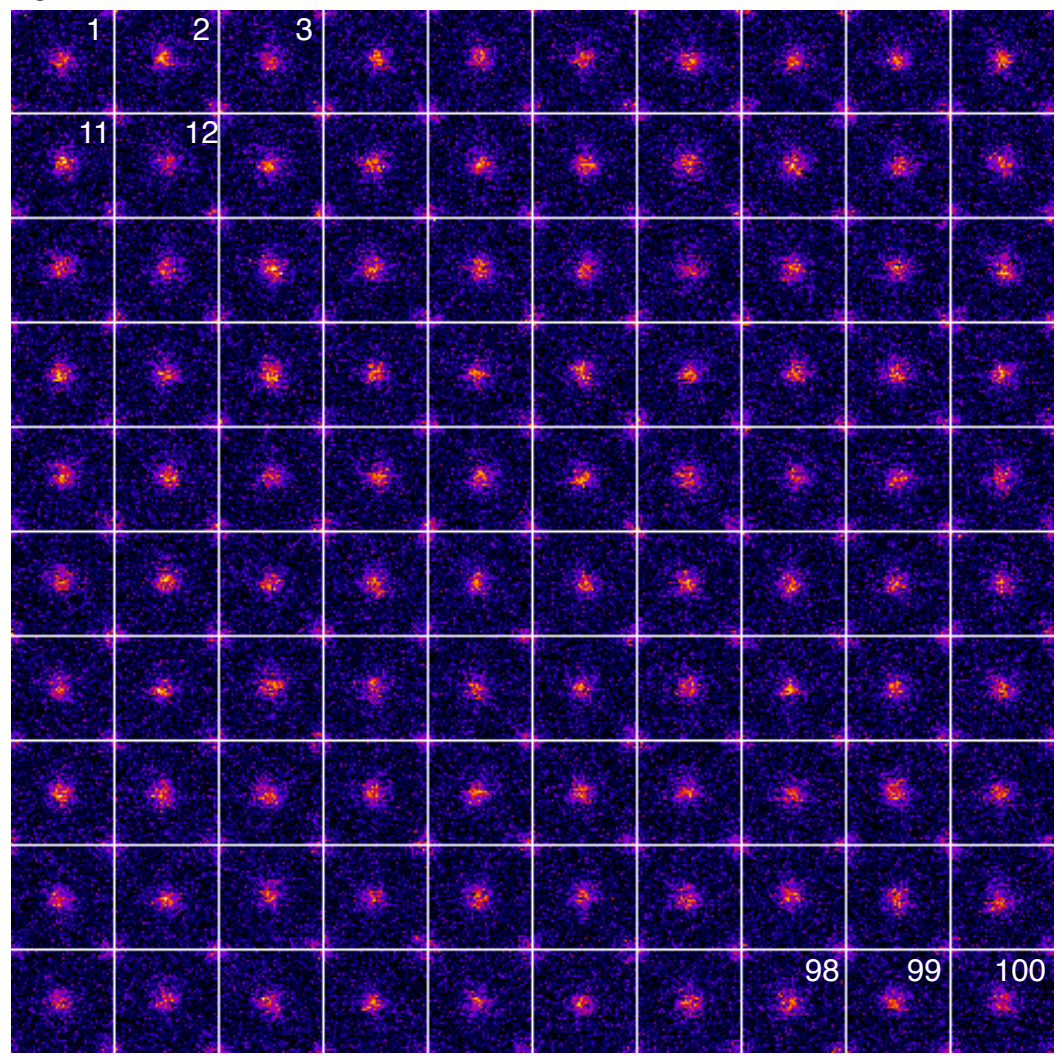

d

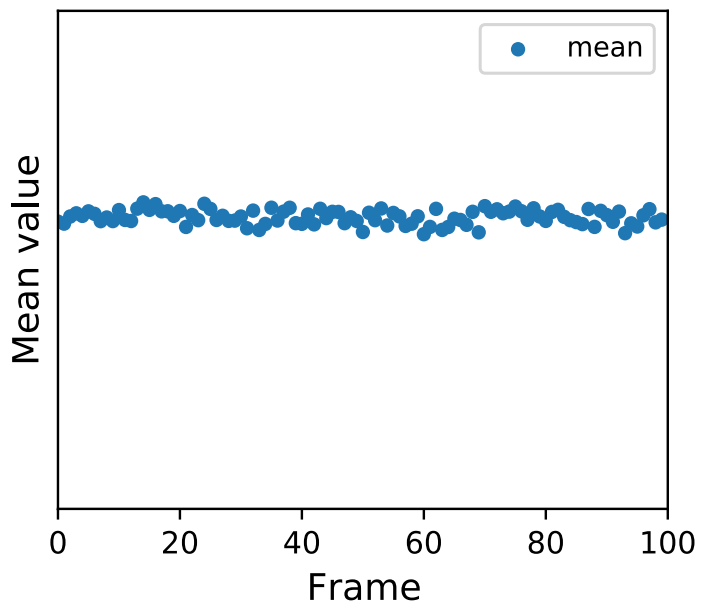

Figure S5. (a) The frame-averaged ADF STEM image obtained from $\mathrm{SrTiO}_{3}$ viewed along the [001] direction, where the images were acquired at a fixed defocus condition (in-focus). (b) The respective frame images of the corresponding $\mathrm{Sr}$ atomic column, as marked in (a). (c) The contrast profile of $\mathrm{Sr}$ atomic column as a function of frame. As a reference, the contrast profile of $\mathrm{Sr}$ atomic column as a function of defocus is given (red profile). (d) The mean profile as a function of frame, obtained from the marked region in (a). The scale bar in (a) is $2 \AA$. 
Supporting Information

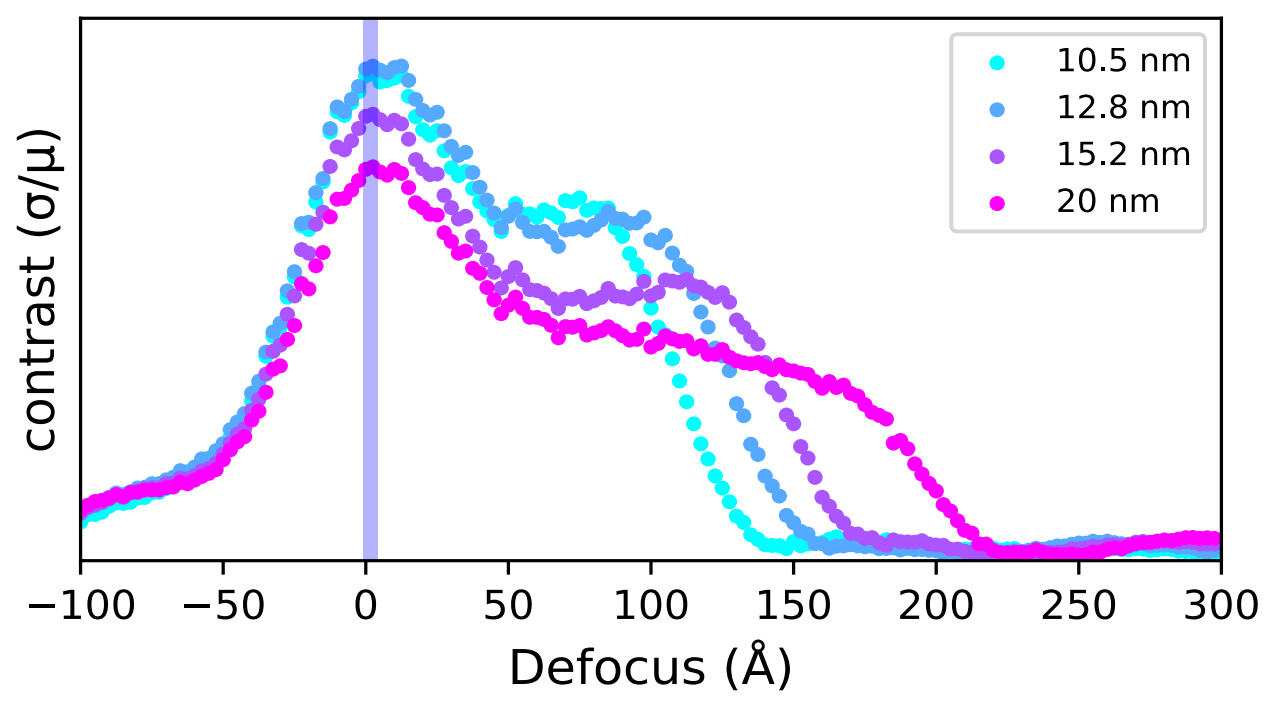

Figure S6. Contrast profile as a function of defocus obtained from $\mathrm{Sr}$ atomic column, where we used 1.5 times larger Debye-Waller factor than that at room temperature. 
Supporting Information
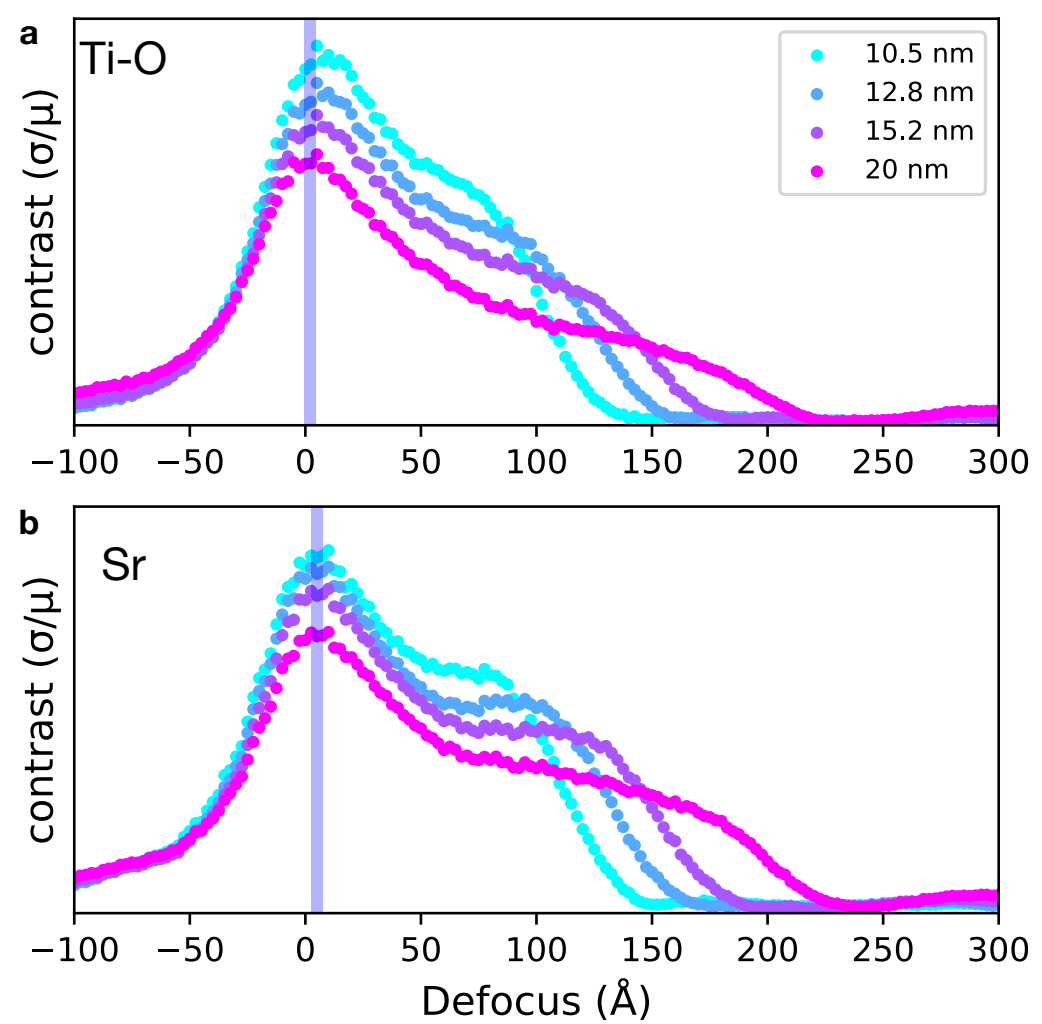

Figure S7. Contrast profiles as a function of defocus obtained from the simulated images with the model of $\mathrm{TiO}_{2}$-layer termination: (a) $\mathrm{Ti}-\mathrm{O}$, (b) $\mathrm{Sr}$ atomic columns, respectively. The assumed specimen thicknesses are $10.5,12.8 \mathrm{~nm}, 15.2 \mathrm{~nm}$ and $20 \mathrm{~nm}$, respectively. 
Supporting Information

a

०.

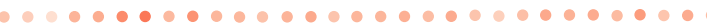
-

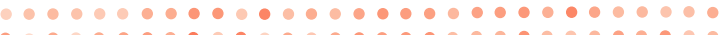
o (

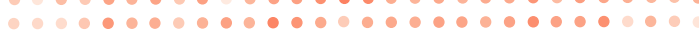

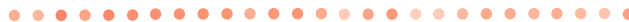
1000

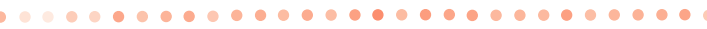
-

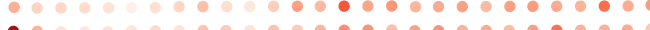
$0-0$ o -

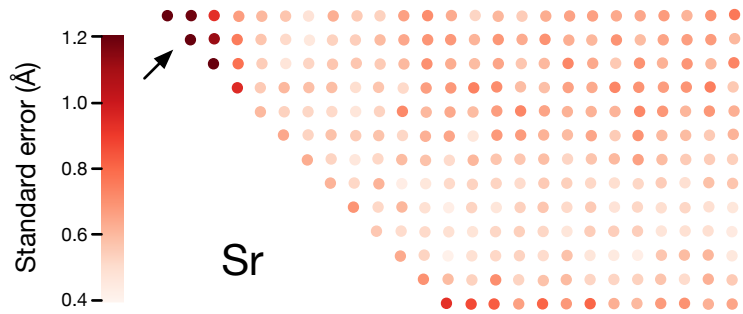

b

,

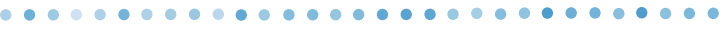
ค

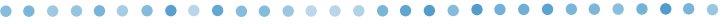
० ० (ब ० ० ०  ०

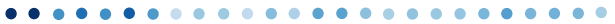

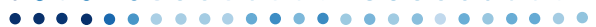

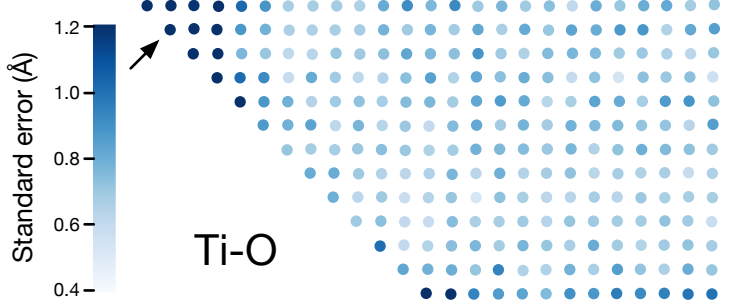

Figure S8. Standard error maps of fitting for (a) Sr, (b) Ti-O atomic columns, respectively. 
Supporting Information

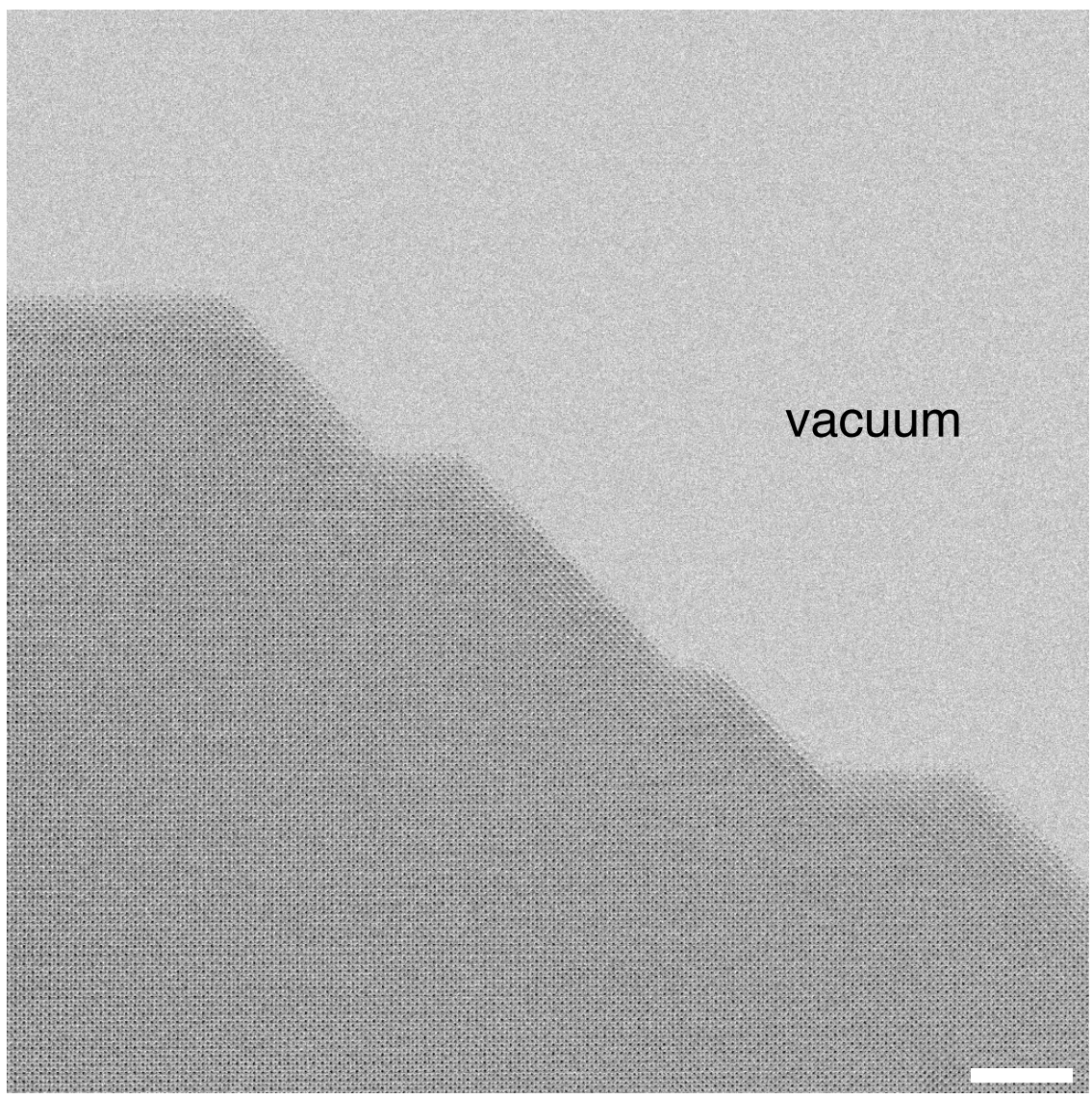

Figure S9. Low-magnified annular bright-field STEM image of $\mathrm{SrTiO}_{3}$ viewed along the [001] direction. The scale bar is $5 \mathrm{~nm}$. 

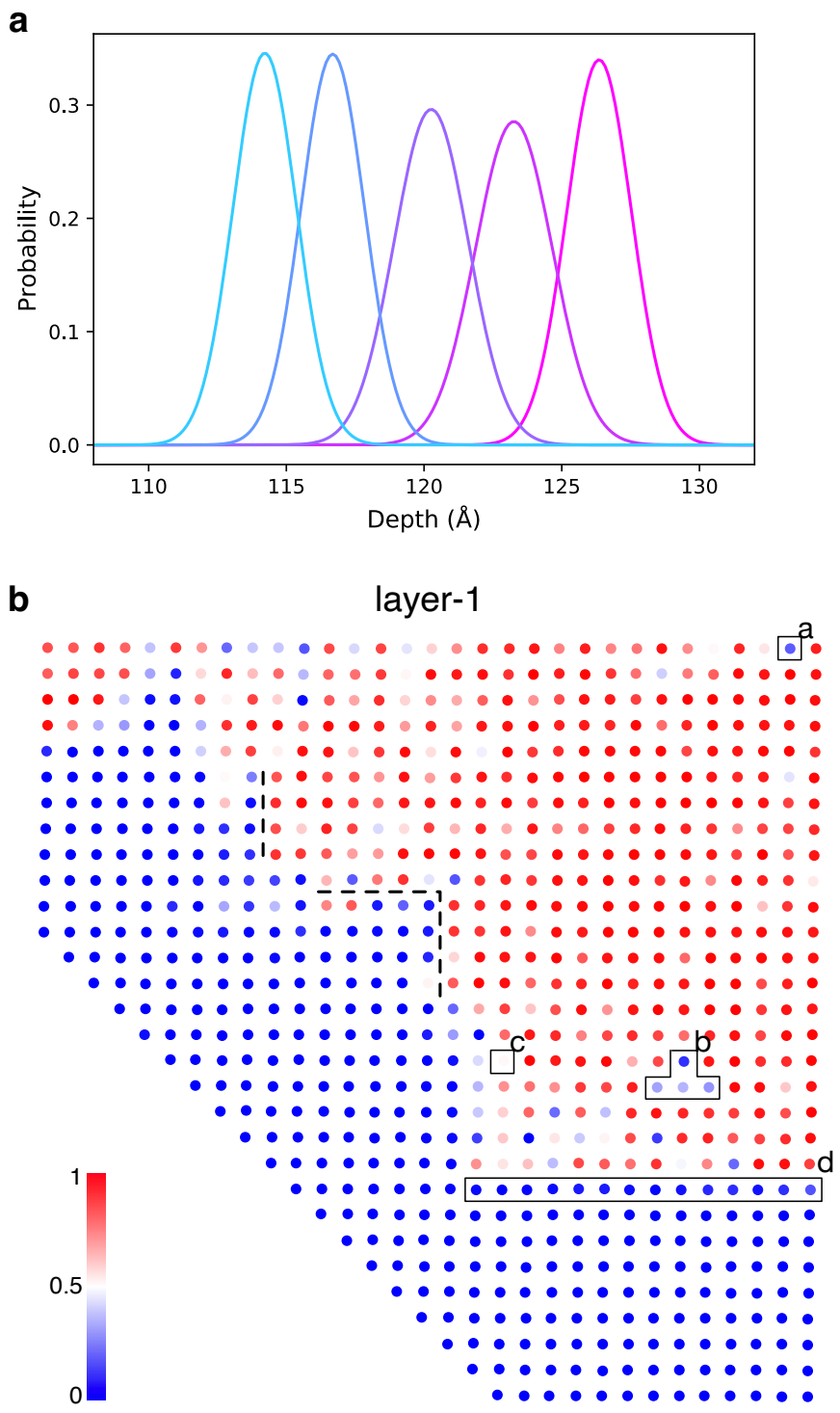

Figure S10. (a) Normalized gaussian profiles obtained from the peak deconvolution in total PDF at $\mathrm{Sr}$ atomic column. (b) The probability map for respective Sr atomic column in layer-1. 
a

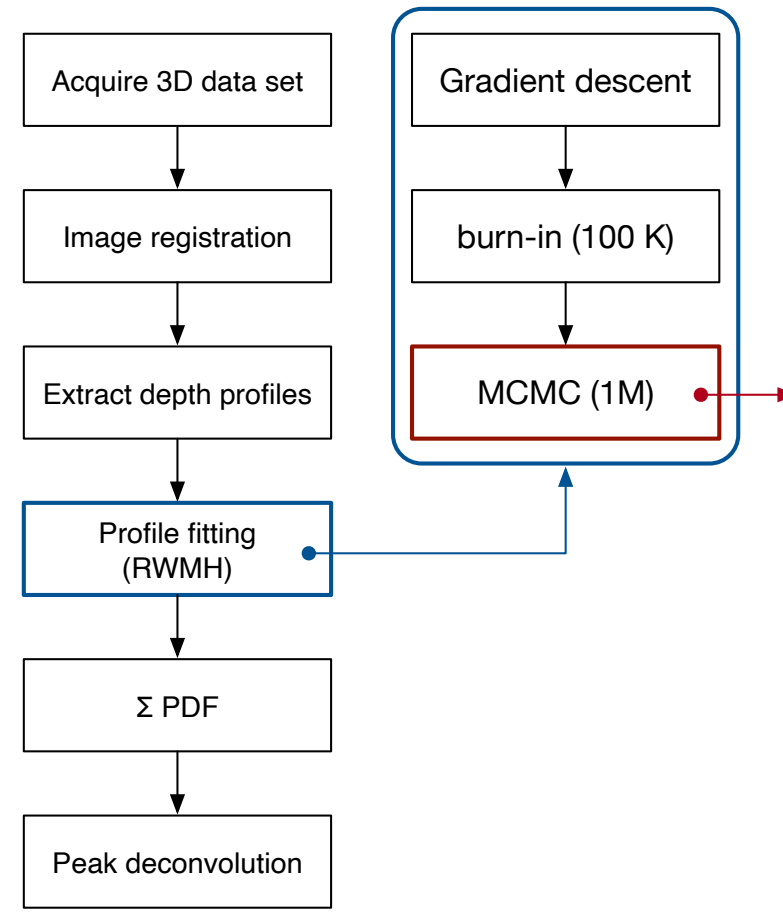

C

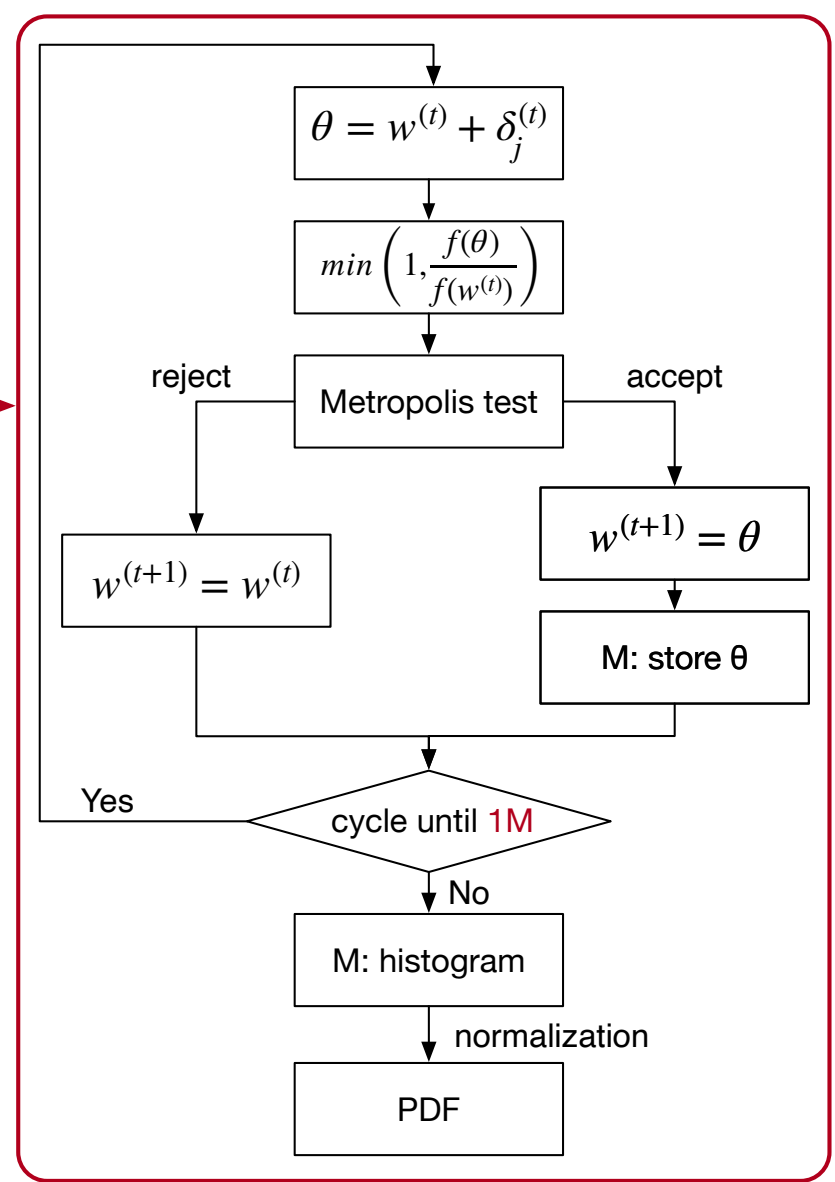

Figure S11. The flowchart of the present analysis, (a) overall flowchart, (b) profile fitting flowchart, and (c) RWMH fitting flowchart. 


\section{REFERENCES}

(1) Ishikawa, R.; Shibata, N.; Taniguchi, T.; Ikuhara, Y. Three-Dimensional Imaging of a Single Dopant in a Crystal. Phys. Rev. Appl. 2020, 13, 034064.

(2) Ishikawa, R.; Pennycook, S. J.; Lupini, A. R.; Findlay, S. D.; Shibata, N.; Ikuhara, Y. Single Atom Visibility in STEM Optical Depth Sectioning. Appl. Phys. Lett. 2016, 109, 163102.

(3) Jones, L.; Yang, H.; Pennycook, T. J.; Marshall, M. S. J.; Van Aert, S.; Browning, N. D.; Castell, M. R.; Nellist, P. D. Smart Align-A New Tool for Robust Non-Rigid Registration of Scanning Microscope Data. Adv. Struct. Chem. Imag. 2015, 1, 1-8.

(4) LeBeau, J. M.; Findlay, S. D.; Allen, L. J.; Stemmer, S. Quantitative Atomic Resolution Scanning Transmission Electron Microscopy. Phys. Rev. Lett. 2008, 100, 206101.

(5) Van Aert, S.; Batenburg, K. J.; Rossell, M. D.; Erni, R.; Van Tendeloo, G. Three-Dimensional Atomic Imaging of Crystalline Nanoparticles. Nature 2011, 470, 374-377.

(6) Ishikawa, R.; Lupini, A. R.; Findlay, S. D.; Pennycook, S. J. Quantitative Annular Dark Field Electron Microscopy Using Single Electron Signals. Microsco. Microanal. 2014, 20, 99-110.

(7) Kirkland, E. J. Advanced Computing in Electron Microscopy; Springer: New York, 2010.

(8) Abramov, Y. A.; Tsirelson, V. G.; Zavodnik, V. E.; Ivanov, S. A.; Brown, I. D. The Chemical Bond and Atomic Displacements in $\mathrm{SrTiO}_{3}$ from X-Ray Diffraction Analysis. Acta Crystallogra. Sec. B 1995, 51, 942-951. 